\title{
Adult Kawasaki Disease Unrelated to Epstein-Barr Virus and Group A Streptococcus
}

\author{
Tomohiro Hattori, Yoshihiro Matsukawa*, Masami TAKeI*, Kenji Yamaguchi, \\ Tetsuo YAMAZAKI*, Umihiko SAWAdA*, Shigemasa SAWAdA*, Takashi HorIE, \\ Mamoru AYUSAWA**, Nobutaka NOTO** and Kensuke HARADA**
}

\begin{abstract}
A 23-year-old Japanese woman with a fever and generalized skin eruptions was referred to our hospital in July 1999. At admission, her temperature was $38.9^{\circ} \mathrm{C}$, and she had fluctuating symptoms including erythema of the extremities, conjunctival hyperemia, strawberry tongue, and generalized skin eruptions, but lymphadenopathy was not verified. An initially elevated urine leukocyte count (more than $\mathbf{1 0 0}$ per high power field) later returned to normal range without antibiotic therapy. Adult Kawasaki disease was diagnosed on the basis of the above symptomology. Echocardiograph showed transient effusion in the pericardium. Using the Harada scoring system for treatment of Kawasaki disease, we gave the patient aspirin and did not administer intravenous immunoglobulin. The clinical course was uneventful, and on the day of discharge (day 22 after onset), the laboratory test results were nearly normal. Laboratory test results were negative for both Epstein-Barr virus and group A Streptococcus.
\end{abstract}

(Internal Medicine 44: 1182-1184, 2005)

Key words: adult, aspirin, Harada scoring system, intravenous immunoglobulin, Kawasaki disease

\section{Introduction}

Kawasaki disease (KD) is an acute febrile vasculitis observed in infants and children (1). KD has also been reported to develop in adults (2-6); however differences in clinical manifestations, epidemiology, and pathoetiology between adults and pediatric forms of the disease have not been in- vestigated. Therefore, when such a disease develops in adults, it could be difficult to establish the correct diagnosis.

Some researchers have reported that Epstein-Barr virus (EBV) plays some roles in the pathogenesis of KD, although serologic evidence for EBV infection may not be found during the disease course $(7,8)$. In addition, group A Streptococcus virulence factors are also considered to be related to KD (9-11). Moreover, no reports have discussed the pathogenic role of EBV and the group A Streptococcus in inducing adult Kawasaki disease (AKD). Hence we report an AKD patient who was negative for the both pathogens.

\section{Case report}

A 23-year-old Japanese woman had a fever $\left(38.0^{\circ} \mathrm{C}\right)$ in July 1999. Generalized skin eruptions developed on day 4 after onset. On the next day, she was admitted to our hospital, her temperature was $39.3^{\circ} \mathrm{C}$, and physical examination revealed hepatomegaly, coated tongue, and erythema of the palms. We could not confirm splenomegaly or lymphadenopathy. Laboratory findings were as follows: C-reactive protein $(\mathrm{CRP}), 16.4 \mathrm{mg} / \mathrm{dl}$; erythrocyte sedimentation rate

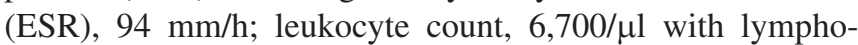
penia $(603 / \mu \mathrm{l})$ and left shift of neutrophils (34\% stab, $47 \%$ segmented, and presence of myelocytes), and $50 \%$ hemolytic unit of complement, $57.9 \mathrm{U} / \mathrm{ml}$. Urinalysis disclosed innumerable leukocytes (more than 100 leukocytes per high power field). Results of serologic tests were not suggestive of acute infection of cytomegalovirus, herpes zoster virus, varicella-zoster virus, adenovirus, measles virus, rubella virus, EBV, and toxoplasma (Table 1). In addition, tests for virulence factors for group A Streptococcus were negative: anti-streptolysin O (ASO) and anti-streptokinase (ASK). Two weeks later, these tests were repeated without significant changes. Bacterial cultures of blood, urine, and tonsil

From Department of Medicine, Division of Respirology, *Department of Medicine, Division of Hematology and Rheumatology and **Department of Pediatrics, Nihon University School of Medicine, Tokyo

Received for publication January 28, 2005; Accepted for publication August 5, 2005

Reprint requests should be addressed to Dr. Yoshihiro Matsukawa, Department of Medicine, Division of Hematology and Rheumatology, Nihon University School of Medicine, Oyaguchi-Kamimachi Itabashi-ku, Tokyo 173-8610 
Table 1. Serologic Tests for Infectious Diseases

\begin{tabular}{llcc}
\hline & & \multicolumn{2}{c}{ Standard values } \\
\hline EBV & & & $10<$ \\
VCA & IgG (FA) & $10<$ & $10<$ \\
& IgM (FA) & $10<$ & $10<$ \\
EA-DR & (FA) & $10<$ & $10<$ \\
EBNA & (FA) & $10<$ & $2.0<$ \\
CMV & IgG (EIA) & $2.0<$ & $0.7<$ \\
& IgM (EIA) & 0.27 & $4<$ \\
Adenovirus & (CF) & $4<$ & $4<$ \\
HSV & (CF) & $4<$ & $4<$ \\
VZV & (CF) & 4 & $4<$ \\
Measles virus & (CF) & $4<$ & $4<$ \\
Rubella virus & (CF) & $4<$ & $5<$ \\
Toxoplasma IgG & (IU/ml) & $3<$ & $0.7<$ \\
Toxoplasma IgM & (IU/ml) & 0.1 & $200<$ \\
ASO & (IU/ml) & 100 & $640<$ \\
ASK & (IU/ml) & 320 & \\
& & & \\
& & &
\end{tabular}

EBV: Epstein-Barr virus, VCA: Viral capsid antigen, EA: Early antigen, EBNA: Epstein-Barr nuclear antigen, CMV: Cytomegalovirus, HSV: Herpes simplex virus, VZV: Varicellazoster virus, ASO: Antistreptolysin-O, ASK: Antistreptokinase.

were negative. Autoantibodies such as anti-nuclear antibody, anti-deoxyribonucleic acid antibody, and anti-ribonucleic protein antibody were negative. On day 3 of hospitalization (day 8 after onset), the patient showed strawberry tongue, conjunctival hyperemia, and heterogeneous erythema on the trunk. Echocardiography showed the presence of mild pericardial effusion on the day 8 after onset, and this effusion resolved 5 days later. At that time, the patient fulfilled five of the diagnostic criteria for $\mathrm{KD}(12): 1$ ) fever lasting more than 5 days; 2 ) erythema and peeling of the skin at the extremities (plate-like desquamation of the palms and soles); 3) heterogenous erythema; 4) conjunctival hyperemia; and 5) strawberry tongue. According to the Harada scoring system $(5,13,14)$, she fulfilled only one criterion (CRP elevation) (Table 2). We thus withheld administration of intravenous immunoglobulin (IVIG) and prescribed aspirin at 1,200 mg/ day beginning on the day 17 after onset. The subsequent clinical course was uneventful. In October 1999 (2 month after the discharge) and October 2000, the patient underwent repeat serologic testing and polymerase chain reaction analysis of blood and oral mucosa samples to check for EBV DNA. Both tests were negative for EBV.

\section{Discussion}

KD usually develops in infants, and only $11 \%$ of reported patients are aged 4 or older (1). When AKD develops in adults, it is not easy to make the correct diagnosis. Hayakawa et al summarized the clinical manifestations of $\mathrm{AKD}$, the general age of onset (mainly between age 20 and
Table 2. Harada Scoring System (Indications* for intravenous gamma-globulin treatment using the during the first 9 days after onset of Kawasaki disease) $(5,13,14)$

\begin{tabular}{lc}
\multicolumn{1}{c}{ Predictor } & Value** \\
\hline 1. White blood cell count & $\geq 12,000 / \mathrm{mm}^{3}$ \\
2. Platelet counts & $<3.5 \times 10^{4} / \mathrm{mm}^{3}$ \\
3. C-reactive protein level & $\geq 4.0 \mathrm{mg} / \mathrm{dl} \mathrm{or} 3+$ \\
4. Hematocrit & $<35 \%$ \\
5. Albumin & $<3.5 \mathrm{~g} / \mathrm{dl}$ \\
6. Age & $\leq 12$ months \\
7. Sex & Male \\
\hline
\end{tabular}

*Intravenous gamma-globulin treatment is indicated if the patient's total score is $\geq 4$ in the acute phase; treatment is not indicated if the score is $\leq 3$ in the acute phase. ${ }^{* *}$ The lowest value for the first 9 days after onset is used. A score of 1 for each category that is positive.

$50)$ and the sex ratio $(\mathrm{M}: \mathrm{F}=2: 1)$, although they did not describe features that discriminate AKD from childhood KD (3). In contrast, Jackson et al reported a relatively high rate of cardiopathy associated with AKD: 3 of $28(10.7 \%)$ of their AKD patients had heart failure or abnormal electrocardiographic findings (4). Such findings have not been reported in childhood KD. The present patient showed cardiopathy involving transient pericardial effusion, which supports the Jackson et al findings. Concerning treatment, we do not have any measures to evaluate the needs for IVIG in AKD patients having cardiopathy. In addition, we did not have any informative experience for the treatment of AKD patients having pericardial effusion. We therefore evaluated the need for IVIG on the basis of the Harada scoring system. Consequently, the effusion and other symptoms ameliorated without IVIG administration reducing treatment costs. We thus presumed that the scoring system was useful in assessing the need for IVIG in AKD patients as well as childhood KD patients $(13,14)$.

The association between EBV and KD has been explored $(7,8)$. There may be an affinity between superantigens presented by EBV and human endothelial cells, the interaction of which induces vasculitis. The evidence of EBV infection is difficult to detect during the course of EBV-related $\mathrm{KD}$ (7, 8). Our patient manifested no evidence of EBV infection throughout the course of illness and has remained negative for any laboratory indications of EBV. In addition, streptococcal pyrogenic exotoxin $\mathrm{C}$, one of the pyrogenic exotoxins (heat-labile toxins) produced by group A Streptococcus, has been reported to act as a superantigen in inducing $\mathrm{KD}$ (15). We however confirmed both ASO and ASK to be negative, and bacterial cultures of the tonsil were negative.

In conclusion, AKD unrelated to both EBV and group A Streptococus developed in a young Japanese woman. Although the Harada scoring system was prepared for the application of IVIG use in patients with pediatric KD irrespective of the presence of coronary aneurysma, it seems useful 


\section{HATTORI et al}

for AKD patients. It might work well for preventing the unnecessary use of IVIG among AKD patients, although more patients are required to assess this issue.

\section{References}

1) Leung DY. Kawasaki disease. Curr Opin Rheumatol 5: 41-50, 1993.

2) Tomiyama J, Hasegawa Y, Kumagai Y, Adachi Y, Karasawa K. Acute febrile mucocutaneous lymph node syndrome (Kawasaki disease) in adults: Case report and review of the literature. Jpn J Med 30: 285-289, 1991.

3) Hayakawa H, Katoh T, Kanda H, Suzuki M, Takahashi S, Numata T. Adult Kawasaki disease associated with transient coronary artery ectasia. Sinzou 28: 814-819, 1996 (in Japanese).

4) Jackson JL, Kunkel MR, Libow L, Gates RH. Adult Kawasaki disease. Report of two cases treated with intravenous gamma globulin. Arch Intern Med 154: 1398-1405, 1994.

5) Saito $\mathrm{T}$, Ishikawa $\mathrm{H}$, Hasunuma $\mathrm{O}$, et al. Report of a case with adult Kawasaki disease. J Nihon Univ Med Assoc 53: 533-538, 1994 (in Japanese, Abstract in English).

6) Vaiopoulos G, Sfifakis PP, Skoumas V, Christov E, Doukas E, Kaklamanis P. Kawasaki's disease in a young adult. Rev Rhum Ed Fr 61: 645-648, 1994 (in French).
7) Iwanaga M, Takada K, Osato T, Saeki Y, Noro S, Sakurada N. Kawasaki disease and Epstein-Barr virus. Lancet 1: 938-939, 1981.

8) Kikuta H, Matsumoto S, Osato T. Kawasaki disease and Epstein-Barr Virus. Acta Paediatr Jpn 33: 765-770, 1991.

9) Barton M, Melbourne R, Morais P, Christie C. Kawasaki syndrome associated with group A streptococcal and Epstein-Barr virus coinfection. Ann Trop Paediatr 22: 257-260, 2002.

10) Cimaz R, Falcini F. An update on Kawasaki disease. Autoimmun Rev 2: 258-263, 2003.

11) Nomura $Y$, Masuda K, Yoshinaga M, Takei S, Miyata K. Probable relationship between streptococcal pyrogenic exotoxin A and Kawasaki syndrome in patients older than six months of age. Pediatr Infect Dis J 22: 794-798, 2003.

12) Rowley AH, Shulman ST. Kawasaki disease. in: Behrman RE, Kliegman RM, Jenson HB, Eds. Nelson's Textbook of Pediatrics, 16th ed. W.B. Philadelphia, Saunders Co., 2000: 725-727.

13) Harada K. Intravenous $\gamma$-globulin treatment in Kawasaki disease. Acta Paediatr Jpn 33: 805-810, 1991.

14) Sato N, Sugimura T, Akagi $T$, et al. Selective high dose gammaglobulin treatment in Kawasaki disease: assessment of clinical aspects and cost effectiveness. Pediatr Int 41: 1-7, 1999.

15) Yoshioka T, Matsutani T, Iwagami $S$, et al. Polyclonal expansion of TCRBV2- and TCRBV6-bearing T cells in patients with Kawasaki disease. Immunology 96: 465-472, 1999. 